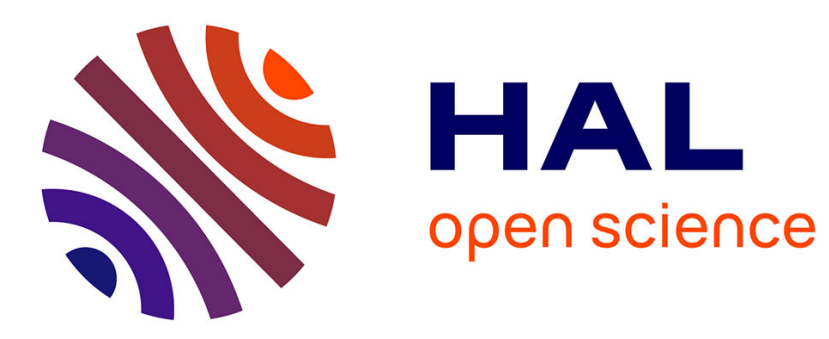

\title{
Reduction of the space-charge field in photorefractive crystals
}

Kimmo Paivasaari, Alexei A. Kamshilin, Philippe Delaye, Gérald Roosen

\section{To cite this version:}

Kimmo Paivasaari, Alexei A. Kamshilin, Philippe Delaye, Gérald Roosen. Reduction of the space-charge field in photorefractive crystals. Optics Communications, 2002, 213, pp.357-366. 10.1016/S0030-4018(02)02082-5 . hal-00686039

\section{HAL Id: hal-00686039 \\ https://hal-iogs.archives-ouvertes.fr/hal-00686039}

Submitted on 6 Apr 2012

HAL is a multi-disciplinary open access archive for the deposit and dissemination of scientific research documents, whether they are published or not. The documents may come from teaching and research institutions in France or abroad, or from public or private research centers.
L'archive ouverte pluridisciplinaire HAL, est destinée au dépôt et à la diffusion de documents scientifiques de niveau recherche, publiés ou non, émanant des établissements d'enseignement et de recherche français ou étrangers, des laboratoires publics ou privés. 


\title{
Reduction of the space charge field in photorefractive crystals
}

\author{
Kimmo Paivasaari and Alexei A. Kamshilin \\ Department of Physics, University of Joensuu, P.O.Box 111, FIN-80101 \\ Joensuu, Finland \\ Philippe Dkaye and Gérald Roosen \\ Laboratoire Charles Fabry de l'Institut d'Optique, Unité Mixte de \\ Recherche 8501 du Centre National de la Recherche Scientifique I \\ Université Paris-Sud, Bat. 503, Centre Scientifique d'Orsay, 91403 \\ Orsay Cedex, France.
}

\begin{abstract}
The space-charge field amplitude inside photorefractive crystals was evaluated using three different experimental techniques: (i) two-wave mixing gain measurements, (ii) direct phase demodulation under external dc-electric field, and (iii) phase demodulation under acfield with proper polarization filtering. The experimental data could be simultaneously fitted with the theory in the frame of the traditional onelevel photorefractive model only if an additional reduction of the space charge field is assumed. The traditional model fails to explain appearance of this reduction factor. Measurements were carried out with $\mathrm{Bi}_{12} \mathrm{TiO}_{20}$ and $\mathrm{GaP}$ photorefractive crystals.
\end{abstract}

\section{Introduction}

Semiconductors and sillenites exhibit strong and fast photorefractive response and therefore are very important as a recording media for adaptive interferometry purposes. Enhancement of light-induced changes of the refractive index can be achieved in these materials by applying an external electric field to the crystal. Temporal form of this external field affects greatly the properties of the grating recording and light interaction inside the crystal. Fields applied to the crystal are typically dc- or square-wave ac-fields. When strong dc-field is applied, the recorded index grating is in phase (or shifted by $\pi$ ) with the illumination pattern while application of ac-field leads to $\pi / 2$ phase shift between the grating and illumination pattern. These two enhancement techniques are often referred to as a local and non-local recording mechanism, respectively. This difference in the phase shift leads to different manifestations of the grating in the wave mixing experiments. Local grating recording enables direct transformation of a fast phase modulation between the interfering beams into an intensity modulation through two-wave mixing inside the crystal [1], although the energy exchange between the beams is negligible. In contrast, non-local grating recording is optimal for the energy exchange between the interfering beams, but the direct phase demodulation is forbidden.

Beside the difference in the phase between the recorded space-charge field and interference pattern there should be differences in the space-charge field amplitude created with different recording techniques. When Stepanov and Petrov 
[2] first introduced the idea of the grating enhancement using ac-field of the square waveform they predicted that the ac-field should produce a larger amplitude by a factor of $p=L_{0} K$ compared to that obtained with dc-field of the same amplitude. $L_{0}$ is the drift length and $K$ is the grating vector. In the literature values as high as $p$ $=4$ are predicted from the theory [3] and achieved in energy exchange experiments [4] with sillenite crystals. However, these results were obtained from coupling gain measurements using ac-field recording and the results were compared only with the theoretical values. Direct comparison of the space-charge field enhancement by applying dc- and ac-field to the crystal has not been yet performed.

By using recently discovered polarization self-modulation (PSM) effect and proper polarization filtering after the crystal, the linear phase demodulation is achieved even with gratings recorded using ac-field enhancement technique [5]. In this paper we perform for the first time, to the best of our knowledge, comparative study on the space-charge field formation using two different phase demodulation techniques in the same sample. Measurements of the amplification gain in the energy exchange experiments were carried out, as well. We have found that all the experimental data can be quite well fitted with the standard theory of the photorefractive effect involving one photo-active level and one type of charge carriers [6]. However, such a fitting is only possible assuming significant reduction of the space charge field in respect with the theoretically predicted magnitude.

Experiments were carried out in sillenite $\mathrm{Bi}_{12} \mathrm{TiO}_{20}$ and semiconductor $\mathrm{GaP}$ samples both in the transversal optical configuration. Changing the input polarization of the interfering beams and installation of the polarization analyzer were the only operations needed between different experiments. The experimental data were compared with both numerical solution of the vectorial system of coupled wave equations [7] and analytical equation for each particular technique.

\section{Theoretical description}

When using the polarization filtering for phase demodulation, the vectorial nature of beam coupling must be taken into account. In photorefractive crystals of cubic symmetry, energy and polarization exchange during light diffraction cannot be separated from each other and the two-wave mixing equations cannot be reduced into the scalar ones. The vectorial theory of wave coupling developed by Sturman et al. [7] can be used to describe all observable properties of the twowave mixing in cubic photorefractive crystals. An analytical expression for the amplitude of the output object and reference beams was derived there assuming that the grating amplitude is uniform along the propagation direction $(m(z)=$ constant). This assumption seems to be justified for the configuration of anisotropic diffraction in which the energy exchange between the interacting beams can be minimized by a proper choice of the input polarization. During fitting the experiment and theory we compare the analytical and numerical solution of coupled wave equations for both modulation techniques. Two-wave mixing equations in the vectorial form neglecting light absorption are [7] 


$$
\begin{aligned}
& \left(\frac{\partial}{\partial z}-i \vec{\kappa} \cdot \hat{\vec{\sigma}}\right) \vec{A}_{1}(z)=i E_{K}\left(v_{0}+\vec{v} \cdot \hat{\vec{\sigma}}\right) \vec{A}_{2}(z) \\
& \left(\frac{\partial}{\partial z}-i \vec{\kappa} \cdot \hat{\vec{\sigma}}\right) \vec{A}_{2}(z)=i E_{K}^{*}\left(v_{0}+\vec{v} \cdot \hat{\vec{\sigma}}\right) \vec{A}_{1}(z)
\end{aligned} .
$$

Here $\vec{A}_{1,2}(z)$ are two-dimensional vectors describing the amplitude and polarization state of the object and reference waves, respectively. The vector $\vec{\kappa}$ defines the linear properties of the light propagation whereas the vector $\vec{v}$ and the scalar $v_{0}$ are responsible for the anisotropic and isotropic light interaction,

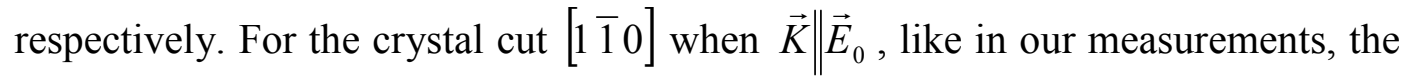
scalar is $v_{0}=0.5 q \cos \xi$ and the vectors get the form

$$
\begin{array}{lll}
v_{1}=q \sin \xi & v_{2}=0 & v_{3}=-0.5 q \cos \xi \\
\kappa_{1}=q E_{0} \sin \xi, & \kappa_{2}=\rho, & \kappa_{3}=-0.5 q E_{0} \cos \xi
\end{array}
$$

where $E_{0}$ is the external electric field, $\vec{K}$ is the wave vector of the recorded grating, $E_{K}$ is the space-charge-field amplitude at the fundamental spatial harmonic (note that it is complex quantity), $\xi$ the angle between the applied field vector and [001] axis, $\rho$ the optical rotatory power and $q=\pi n_{0}^{3} r_{41} / \lambda, n_{0}$ is the refractive index, $r_{41}$ electro-optic coefficient and $\lambda$ is the wavelength. The sigma matrices are given

$$
\hat{\sigma}_{1}=\left(\begin{array}{ll}
0 & 1 \\
1 & 0
\end{array}\right), \quad \hat{\sigma}_{2}=\left(\begin{array}{cc}
0 & -i \\
i & 0
\end{array}\right), \quad \hat{\sigma}_{3}=\left(\begin{array}{cc}
1 & 0 \\
0 & -1
\end{array}\right) .
$$

With the help of the mathematical properties of the sigma matrices, the solution of the Eqs.1 with matrix exponential functions can be reduced to the linear functions resulting in following amplitude for the object beam transmitted through the crystal assuming $m(z)=$ constant:

$$
\vec{A}_{1}(L)=\hat{T}_{+}(L) \vec{A}_{1}(0)+\exp \left(i\left(\phi_{0}+\varphi\right)\right) \hat{T}_{-}(L) \vec{A}_{2}(0)
$$

where $\hat{T}_{ \pm}$are $2 \times 2$ transformation matrices, $\phi_{0}$ is the constant phase shift between the grating and the interference pattern, $\varphi$ is the transient phase shift introduced between the interfering beams and $L$ is the length of the crystal. The transformation matrices are defined as [7]

$$
\begin{gathered}
2 \hat{T}_{ \pm}(L)=\exp \left(i \hat{g}_{+} L\right) \pm \exp \left(i \hat{g}_{-} L\right), \\
\left.\exp \left(i \hat{g}_{ \pm} L\right)=\exp \left( \pm i v_{0}\left|E_{K}\right| L\right) \mid \cos \left(|\vec{\kappa} \pm \vec{v}| E_{K} \| L\right)+i\left(\vec{n}_{ \pm} \cdot \hat{\vec{\sigma}}\right) \sin \left(|\vec{\kappa} \pm \vec{v}| E_{K} \| L\right)\right],
\end{gathered}
$$

where $\vec{n}_{ \pm}=\vec{\kappa} \pm \vec{v}\left|E_{K}\right| /|\vec{\kappa} \pm \vec{v}| E_{K}||$ and $\vec{n} \cdot \hat{\vec{\sigma}}=\left(n_{1} \hat{\sigma}_{1}+n_{2} \hat{\sigma}_{2}+n_{3} \hat{\sigma}_{3}\right)$. 
To achieve the linear phase-to intensity transformation under external acfield $\left(\phi_{0}=\pi / 2\right)$, a polarization analyzer must be installed after the crystal [8]. The light beam intensity after the crystal-analyzer pair can be calculated with the equation

$$
I_{1}^{a c}(L, \varphi)=\mid \sqrt{I_{10}}\left(\vec{e}_{0} \cdot \hat{T}_{+}(L) \cdot \vec{e}_{1}\right)+i \exp (i \varphi) \sqrt{I_{20}}\left(\vec{e}_{0} \cdot \hat{T}_{-}(L) \cdot \vec{e}_{1}\right)^{2},
$$

where $\vec{e}_{1}, \vec{e}_{0}$ are normalized Jones vectors for the input polarization and polarization analyzer, respectively. $I_{10}=\left|A_{1}(0)\right|^{2}$ and $I_{20}=\left|A_{2}(0)\right|^{2}$ are intensities of the interacting beams at the input of the crystal.

A convenient parameter for comparison of the experiment and theory is a fractional intensity modulation defined as an output intensity change caused by the transient phase shift $\varphi$ divided by an average output intensity, $\left[I_{1}(L, \varphi)-I_{1}(L, 0)\right] / I_{1}(L, 0)$. According with Eq.1, the key parameter affecting the fractional intensity modulation is the amplitude of the space-charge field grating, $E_{K}$. Consequently, one can estimate the space-charge field from the measured data of the fractional intensity modulation. This method of space-charge-field evaluation is also known in the literature as a grating translation technique [9].

The space-charge field is a function of the external electric field and it naturally depends on the grating enhancement technique. In the frame of the standard one-level model of the photorefractive effect [6] and in the linear approximation $(m<<1)$, an analytical expression for the amplitude $E_{K}$ can be derived for the case when a fast-oscillating ac-field of the square-wave form is applied to the crystal $[3,7]$.

$$
E_{K}=-i \frac{m}{2} E_{q} \frac{E_{0}{ }^{2}+\left(E_{D}+E_{M}\right) E_{D}}{E_{0}{ }^{2}+\left(E_{q}+E_{D}\right)\left(E_{D}+E_{M}\right)} .
$$

Here the characteristic fields are defined as $E_{D}=k_{b} T K / e, E_{q}=e N_{e f f} /\left(\varepsilon \varepsilon_{0} K\right)$ and $E_{M}=1 /(\mu \tau K)$ with the grating vector modulus of $K=2 \pi / \Lambda$ and the grating period of $\Lambda . N_{\text {eff }}$ is the effective trap concentration and $\mu \tau$ is the mobility-lifetime product of the charge carriers. The modulation index of the intensity pattern is a complex quantity and it depends on the propagation coordinate $z$ :

$$
m(z)=\frac{2 A_{1}(z) A_{2}^{*}(z)}{\left|A_{1}(z)\right|^{2}+\left|A_{2}(z)\right|^{2}} .
$$

Calculating the output intensity modulation using the analytical expression of Eq.7, we introduce the space charge field with invariable modulation index of $m(0)$. In contrast, rigorous solution of coupled wave equations (Eq.1) accounts for variations of the index modulation caused by both energy and polarization exchange. In the case of ac-enhancement, the space-charge field is always purely imaginary (Eq.9) that means independence of the phase shift between the index grating and interference pattern $\left(\phi_{0}=\pi / 2\right)$ on the applied field $E_{0}$. In the transverse configuration, the highest rate of the phase-to intensity transformation is achieved 
when the input linear polarization either parallel or orthogonal to the electric field vector and the output analyzer is either closed or open [8].

Condition of $\phi_{0}=\pi / 2$ is optimal for the light beam amplification and the largest gain in the transverse configuration is achieved when the input polarization makes an angle of $45^{\circ}$ to the electric field vector. In this case the exponential gain factor $\Gamma$ is

$$
\Gamma=q E_{q} \frac{E_{0}^{2}+\left(E_{D}+E_{M}\right) E_{D}}{E_{0}^{2}+\left(E_{q}+E_{D}\right)\left(E_{D}+E_{M}\right)} .
$$

This equation will be used for fitting of the experimental data of the light energy exchange.

When the grating is formed under dc-field, its amplitude within the same approximations of small $m$ is given by [6]

$$
E_{K}(z)=-\frac{m(z)}{2} \frac{E_{q}\left(E_{0}+i E_{D}\right)}{E_{q}+E_{D}-i E_{0}} .
$$

Note that only the real part of the space-charge field is responsible for the linear phase demodulation in the case of the dc-enhancement. As seen from Eq.10, the real part of $E_{K}$, and consequently the phase shift $\phi_{0}$, depends on the applied field $E_{0}$ that must be taken into account while fitting the experimental data. To achieve the highest rate of the phase-to-intensity transformation in the transverse configuration, the input polarization state should be linear making an angle of $45^{\circ}$ with the axis [001] (in crystals without optical activity). In this case the polarization eigenmodes are excited and the analytical expression for the output intensity of the phase-modulated beam takes the form [10]:

$$
I_{1}^{d c}(L, \varphi)=I_{10}[\exp (2 \operatorname{Im}(\gamma) L)+2 \exp (\operatorname{Im}(\gamma) L) \sin (\operatorname{Re}(\gamma) L) \sin (\varphi)]
$$

with

$$
\gamma=-q \frac{E_{q}\left(E_{0}+i E_{D}\right)}{E_{q}+E_{D}-i E_{0}} .
$$

Equations $(12,13)$ were used to fit the data of direct phase demodulation experiment. Moreover, these were compared with the rigorous solution of the coupled wave equations using the space charge field of Eq.10 that takes into account the optical activity of the crystal, as well.

\section{Methodology of the experiment}

Experiments were carried out in a typical two-wave mixing configuration shown in Fig.1. Two mutually coherent beams were derived from laser by means of a beam-splitter. The object beam was reflected from a plain mirror attached to a loudspeaker. The phase of the object beam was modulated at a frequency higher than the inverse response time of the crystal with the amplitude much smaller than 
the wavelength. The grating recording process is not supposed to be affected by such a phase modulation. The object and reference beams of the same linear polarization were made to intersect inside a photorefractive sample forming an interference pattern. The transmitted object beam was collected directly to a photodiode when dc-field is applied to the crystal, but filtered with polarization analyzer before being collected to the photodiode when ac-field is applied. The experimental conditions throughout the measurements were exactly the same for both enhancement techniques except for different input polarization and need of the polarization filtering when applying ac-field.

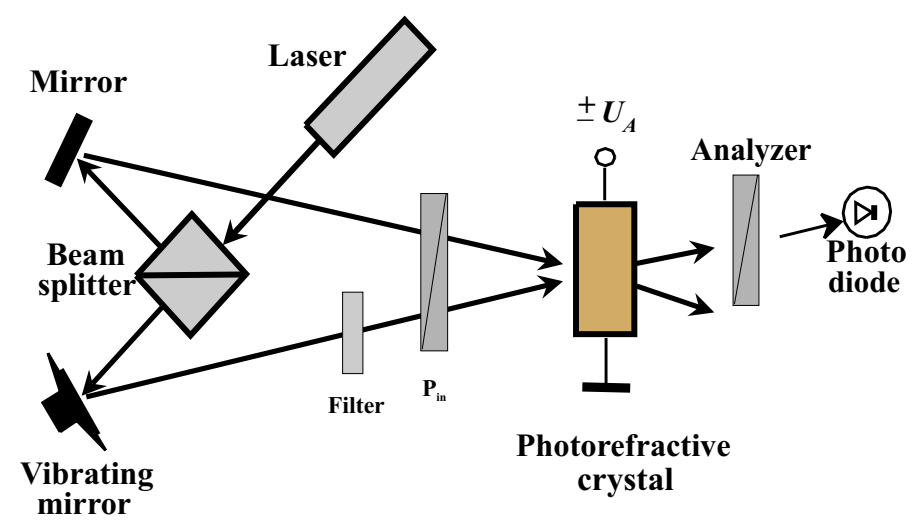

Figure 1. Two-wave mixing set-up used in the measurements.

Two different photorefractive crystals of the same crystallographic cut were studied. Semiconductor GaP sample ( $\overline{4} 3 \mathrm{~m}$ symmetry group) has size of 5.7 $\mathrm{mm}$ along $\langle 001\rangle, 3.79 \mathrm{~mm}$ along $\langle 1 \overline{1} 0\rangle$, and $6.59 \mathrm{~mm}$ along $\langle 110\rangle$ crystallographic axis while sillenite $\mathrm{Bi}_{12} \mathrm{TiO}_{20}$ (BTO) sample (23 symmetry group) has size of $6.73 \mathrm{~mm}, 1.66 \mathrm{~mm}$ and $1.33 \mathrm{~mm}$ along the same axes, respectively. In both samples, the external electric field was applied along $\langle 1 \overline{1} 0\rangle$ axis and the grating vector $\vec{K}$ was parallel to the electric field. A laser diode emitting at the wavelength of $850 \mathrm{~nm}$ and a He-Ne laser at $633 \mathrm{~nm}$ were used with $\mathrm{GaP}$ and BTO samples, respectively. The light beams were expanded to cover whole input face of samples in order to minimize external field screening. Our high-voltage generator produces both dc- and ac-voltage of the square waveform. The period $T$ of ac-voltage was chosen to satisfy the condition of $\tau<<T<<\tau_{g}$ for stationary grating recording, where $\tau$ is the charge-carrier lifetime and $\tau_{g}$ is the grating response time. The phase of the object beam was harmonically modulated at the frequency higher than $\tau_{g}^{-1}$ with the amplitude of 0.24 radians for BTO sample and 0.47 radians for GaP sample.

In both phase demodulation experiments (under dc- and ac- electric field), the fractional intensity modulation, $\left[I_{1}(L \varphi)-I_{1}(L, 0)\right] / I_{1}(L, 0)$, defined in the previous section was measured as a function of the applied field. The energy exchange experiments were carried out applying ac-field to a crystal and measuring the intensity of the transmitted object beam with and without reference also as a function of the external field. From these data we calculated the exponential gain $\Gamma$ using known expression [11] 


$$
\gamma=\frac{(\beta+1) \exp (\Gamma L)}{\beta+\exp (\Gamma L)}
$$

where $\gamma$ is the ratio of the object beam measured with and without reference beam and $\beta=I_{20} / I_{10}$ is the input intensity ratio of the reference and object beams.

To carry out different measurements in similar experimental conditions, the following procedure was chosen. First, we adjusted the input polarization of both beams in order to maximize phase demodulation. The optimal input polarization in the absence of the optical activity is along the crystal eigenaxes for dc-field measurements. For ac-field measurement the optimal condition is achieved when input polarization excite both eigenaxes equally. In the presence of optical activity the optimal input angles are slightly different and can be found for ac-field by minimizing the enhancement coefficient $\gamma$ under the strongest ac-field. Then we introduced phase modulation into the object beam, installed the polarization analyzer after the crystal, and adjusted transmission of the analyzer to achieve the linear phase-to-intensity transformation. The input intensity ratio $\beta$ was set by means of conventional optical filters. Both the intensity modulation caused by the phase modulation $\varphi$ and the average intensity were measured by a photo-diode connected with the oscilloscope during one semi-cycle of the external field as a function of the ac-field amplitude. Then we rotated the input polarization by $45^{\circ}$ and removed the analyzer for the dc-field measurement. Input intensity ratio was adjusted to be the same as during ac-field measurements. Varying dcfield, we measured again the dependence of the fractional intensity modulation on the external dc-field. Thereafter, using the same input polarization as in the dcfield experiment but switching off the phase modulation, the enhancement coefficient $\gamma$ was measured as a function of the ac-field. Series of these measurements were repeated after changing the crossing angle between object and reference beams (changing the grating spacing).

As it follows from the theoretical consideration, all our measurable parameters primarily depend on the product $n_{0}^{3} r_{41} E_{0}$, which we have measured each time after any re-arrangement of the optical set-up using the convenient ellipsometric technique. Since the period of the ac-field was much smaller than the dielectric relaxation time of a crystal, the field screening effect is minimized and $E_{0}=U_{0} / d$, where $U_{0}$ is applied voltage and $d$ is the inter-electrode distance. In this case, the ellipsometric measurements allow us to calculate not only $n_{0}^{3} r_{41}$ but the exact orientation of the sample as well [8]. When dc-field is applied to a sample, ellipsometric measurements reveal an effective electro-optic coefficient taking into account the possible screening effect. Experimentally obtained data of the electro-optic figure of merits were used for fitting of the phase-demodulation and gain experiments with the theory.

\section{Experimental results and their fitting}

First we show data obtained with the direct phase demodulation technique using the BTO sample. Figure 2 shows the fractional intensity modulation as a function of the applied dc-electric field measured at three different grating spacing. In spite of relatively good uniform illumination of the crystal, ellipsometric measurements revealed that the effective electrooptic coefficient 
depends on the setup arrangement. We found that $r_{\text {eff }}=3.4,3.6$, and $4.2 \mathrm{pm} / \mathrm{V}$ for the grating spacing of 2,6 , and $12 \mu \mathrm{m}$, respectively. Note that there were no electric field screening in the experiments with ac-field leading to $r_{41}=5.0 \mathrm{pm} / \mathrm{V}$ at any grating spacing. It is seen that the fractional intensity modulation exhibits linear dependence on the external dc-field in accordance with predictions of both analytical expression of Eq.12 and numerical solution of Eq.1 when Eq.11 expresses the space charge field. There is also slight dependence on the grating spacing due to the saturation field $E_{q}$, which is a function of $K$. However, direct using of Eq.11 (or Eq.13) results in the gradient of all curves being much higher than we observe in the experiment. Only after introducing an additional reduction factor $f_{E}=0.56$ in Eq.11, we were able to fit all the data simultaneously. It is worth noting that even higher reduction of the space charge field was observed in other crystals in similar direct-phase-demodulation experiment [12]. Moreover, this reduction depends on the used wavelength [12]. Note that the space charge field does not depend on the mobility-lifetime product when it is recorded under dcfield. We will discuss possible reasons of the space-charge-field reduction in the next section.

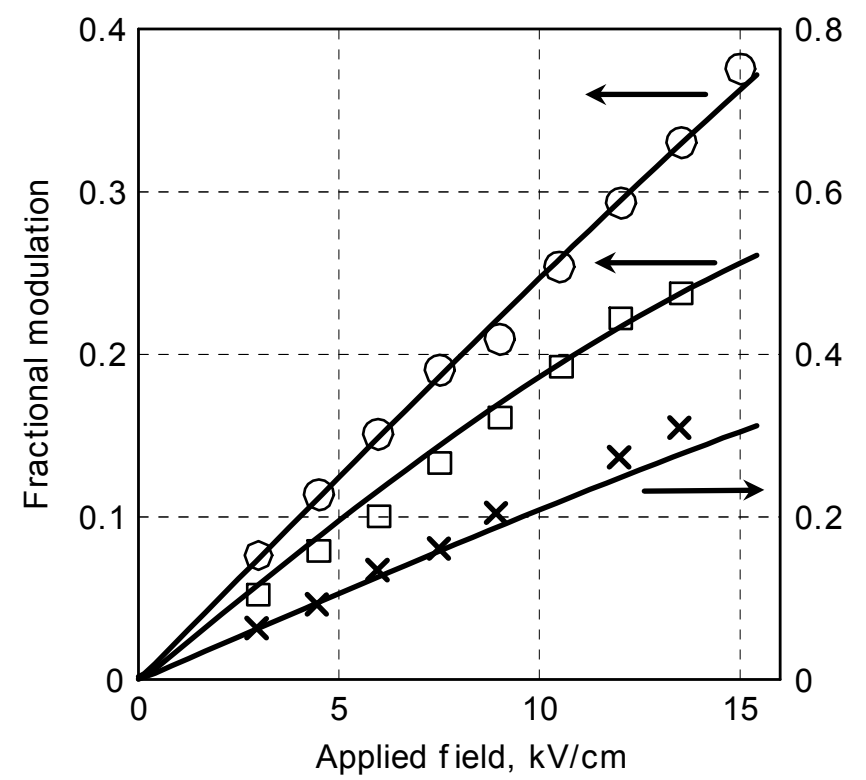

Figure 2. Measured phase demodulation signal using dc-field recording with BTO sample at the grating of $2 \mu \mathrm{m}$ (squares), $6 \mu \mathrm{m}$ (crosses) and $12 \mu \mathrm{m}$ (circles). The solid lines are theoretical curves calculated using Eq. 1 with $E_{K}$ given by Eq.11 but with the reduction factor of 0.56 . For clearer presentation the scale of the dependence at $6 \mu \mathrm{m}$ is different (right-hand side).

Second we present the experimental data of the signal beam amplification obtained with the same BTO sample under external ac-field. Figure 3 shows twowave mixing gain $\Gamma$ as a function of the electric field measured at the grating spacing of $4 \mu \mathrm{m}$ with the input intensity modulation index $m(0)=0.1$ (the intensity ratio $\beta=400$ ). Such type of measurements is frequently used for estimation of both the effective concentration of traps, $N_{\text {eff }}$, and mobility-lifetime product of charge carriers, $\mu \tau$. The solid line in Fig. 3 is the theoretical dependence calculated using Eq.10 with $N_{\text {eff }}=4.6 \times 10^{16} \mathrm{~cm}^{-3}$ and $\mu \tau=2 \times 10^{-8} \mathrm{~cm}^{2} / \mathrm{V}$. Note that the same reduction factor $f_{E}=0.56$ was applied here to $E_{K}$ during the fitting. We found that 
the analytical dependence of Eq.10 is undistinguishable from the numerical solution of Eq. 1 using $E_{K}$ of Eq. 8 although Eq.10 does not take into account the optical activity of the crystal. In fact, the experimental data of Fig. 3 could also be well fitted without the reduction factor but such a fitting leads to significantly different magnitude of $N_{\text {eff }}$ and $\mu \tau$. Deduced traps concentration of $4.6 \times 10^{16} \mathrm{~cm}^{-3}$ was used for fitting of data measured under dc-field (Fig.2).

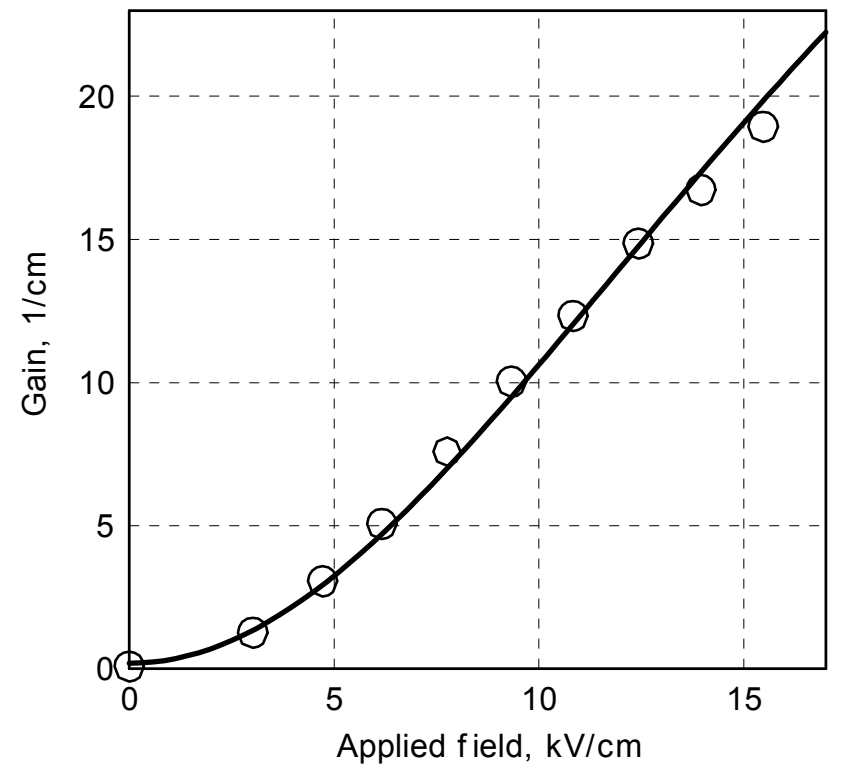

Figure 3. Two-wave mixing gain as a function of the applied field for the BTO sample (circles). Solid line is the theoretical dependence of Eq. 10 calculated with $N_{\text {eff }}=4.6 \cdot 10^{16}$ $\mathrm{cm}^{-3}$ and $\mu \tau=7.6 \cdot 10^{-13} \mathrm{~m}^{2} /$ VThe reduction factor of 0.56 was introduced to $E_{K}$ given by Eq.10. The grating spacing of $4 \mu \mathrm{m}$ and the modulation index $m(0)=0.1$ were used in the measurement.

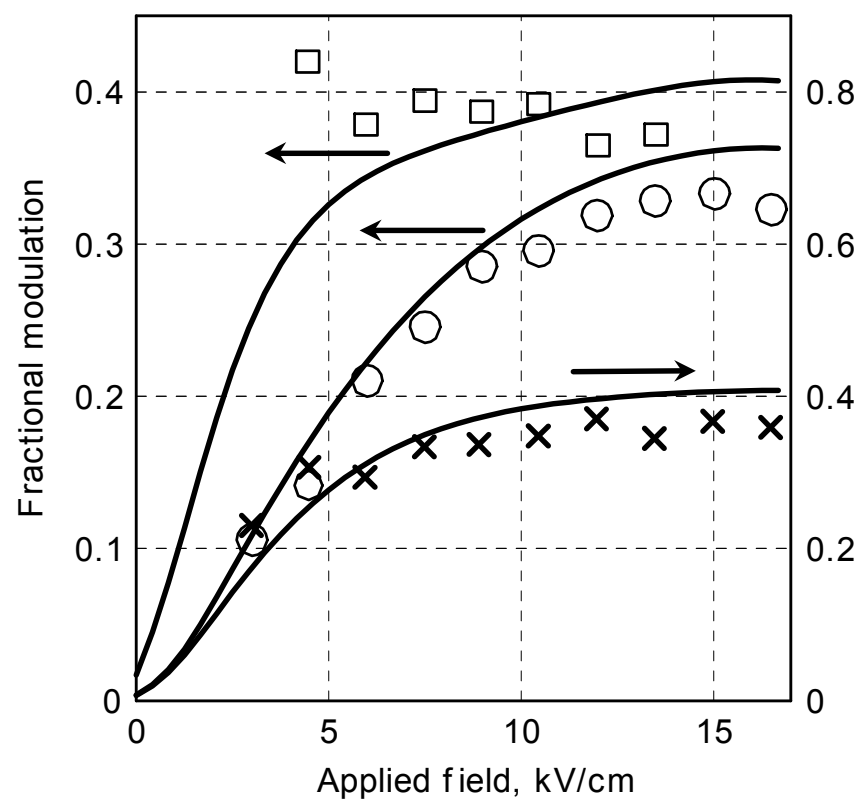

Figure 4. Fractional intensity modulation measured with the PSM technique in the BTO sample. The same set of parameters as in Figures 2,3 (including the reduction factor of 0.56 ) was used for fitting with the numerical solution of Eq.1 shown by solid lines. Grating spacing of $2 \mu \mathrm{m}$ (squares), $6 \mu \mathrm{m}$ (crosses) and $12 \mu \mathrm{m}$ (circles) were used. 
For clearer presentation the scale of the dependence at $6 \mu \mathrm{m}$ is different (right-hand side).

Next we show in Fig.4 the fractional intensity modulation as a function of the electric field measured with the same BTO sample under ac-electric field and proper output polarization filtering. The measurements were carried out at the same grating spacing as in the case of the direct phase demodulation technique and with the same input intensity modulation index $m(0)=0.1$. Solid lines in Fig.4 are the theoretical curves calculated with Eq. 1 and the space charge field given by Eqs.8,9 with $N_{e f f}=4.6 \times 10^{16} \mathrm{~cm}^{-3}$ and $\mu \tau=2 \times 10^{-8} \mathrm{~cm}^{2} / \mathrm{V}$ obtained from the gain experiment. It should be underlined that we also applied the same space-chargefield reduction factor of 0.56 while fitting the data. All curves were fitted simultaneously. One can see a reasonable agreement of the experiment and theory in Fig.4. Since the PCM technique deals with the amplitude of light, the theoretical dependences are very sensitive to small changes of the material parameters of $N_{\text {eff }}$ and $\mu \tau$. Nevertheless, we succeeded to fit the data from three different experiments using the same set of material parameters. Note that simultaneous fitting of all experimental data is impossible without introduction of the space-charge-field reduction factor.

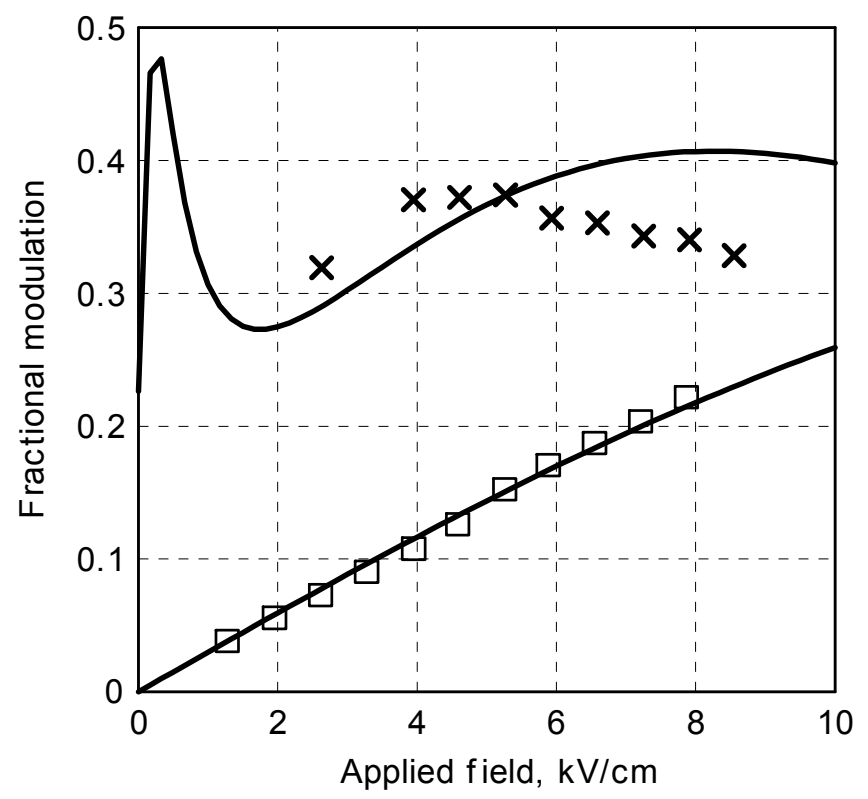

Figure 5. Fractional intensity modulation measured with the GaP sample under ac(crosses) and dc-field (squares). Both curves were fitted simultaneously using the reduction factor for the space charge field of 0.28 . Grating spacing of $3.6 \mu \mathrm{m}$ and modulation index $m(0)=0.1$ for dc-field and $m(0)=0.4$ for ac-field were used.

Similar experiments except the measurement of the amplification gain (which was rather small) was carried out with the GaP sample at the wavelength of $0.85 \mu \mathrm{m}$. The results are shown in Fig.5, where crosses correspond to the phase demodulation using PSM-technique under ac-field and squares are direct phase demodulation under dc-field. Solid lines in Fig.5 show are theoretical dependences calculated using $N_{\text {eff }}=2.1 \times 10^{15} \mathrm{~cm}^{-3}$ and $\mu \tau=1 \times 10^{-8} \mathrm{~cm}^{2} / \mathrm{V}$ for both techniques. Here the reduction of the space charge field is stronger than for BTO: $f_{E}=0.28$. One can see that the theory quite satisfactory fits the experiment in the case of the $\mathrm{GaP}$ sample, as well. A peak of the fractional modulation at small voltages in the 
theoretical dependence for the PSM-technique is hardly observable in the experiment because we exploit the configuration with the closed analyzer that results in the low average output intensity at small dc-field. Note that the reduction factor of the same GaP sample was reported to be much smaller at the wavelength of $0.6328 \mu \mathrm{m}$ [12]. We also observed here the screening effect of the dc-electric field resulting in the effective electrooptic coefficient of $0.6 \mathrm{pm} / \mathrm{V}$ compared to $0.63 \mathrm{pm} / \mathrm{V}$ measured under ac-field. This difference was taken into account while fitting the data.

It is worth noting that both crystals exhibit unusual dependence of the fraction intensity modulation on the modulation index $m(0)$ of the input interference pattern. The standard photorefractive model predicts the saturation of $E_{K}$ when $m \rightarrow 1$ due to formation of higher spatial harmonics. Consequently, the fractional intensity modulation should be also a nonlinear function of $m$. However, we found that this dependence is fairly linear varying $m$ from 0.06 to 1 for the BTO sample and from 0.1 to 1 for the GaP sample. Such an unusual behavior can be due to reduction of the space charge field.

The magnitudes of the effective trap density and mobility-lifetime product obtained from fitting our data with the theory are within the wide range of reported values for BTO crystals. The effective trap concentration of $2 \times 10^{16} \mathrm{~cm}^{-3}$ and $3 \times 10^{16} \mathrm{~cm}^{-3}$ and the mobility-lifetime product of $2 \times 10^{-9} \mathrm{~cm}^{2} / \mathrm{V}$ and $6.8 \cdot 10^{-8} \mathrm{~cm}^{2} / \mathrm{V}$ are given in the Refs. 7 and 4 , respectively. However, value of the mobilitylifetime product as small as $3 \times 10^{-10} \mathrm{~cm}^{2} / \mathrm{V}$ was also reported for BTO [13]. For GaP there are only few reported results. $N_{\text {eff }}$ of $5.5 \times 10^{14} \mathrm{~cm}^{-3}$ and $\mu \tau$ of $1.7 \times 10^{-8}$ $\mathrm{cm}^{2} / \mathrm{V}$ measured in semi-insulating GaP are given in [14]. The parameters used in our calculation and obtained from the measurements are listed in the Table 1. We believe that our method of simultaneous fitting of the data from three different experiments provides the highest fidelity for estimation of the material parameters.

Table 1. Parameters used in the calculations and obtained from phase demodulation measurements.

\begin{tabular}{|c|c|c|}
\hline Parameters & BTO & GaP \\
\hline Rotatory power, $\rho$ & $113 \mathrm{rad} / \mathrm{m}$ & 0 \\
\hline Dielectric constant & 47 & 11 \\
\hline $\begin{array}{l}\text { Effective electro-optic } \\
\text { coefficient, } r_{\text {eff }}\end{array}$ & $5.0 \cdot 10^{-12} \mathrm{~m} / \mathrm{V}$ & $0.63 \cdot 10^{-12} \mathrm{~m} / \mathrm{V}$ \\
\hline $\begin{array}{l}\text { Mobility-lifetime } \\
\text { product, } \mu \tau\end{array}$ & $2 \cdot 10^{-12} \mathrm{~m}^{2} / \mathrm{V}$ & $1 \cdot 10^{-12} \mathrm{~m}^{2} / \mathrm{V}$ \\
\hline $\begin{array}{l}\text { Effective trap } \\
\text { Density, } N_{e f f}\end{array}$ & $4.6 \cdot 10^{22} \mathrm{~m}^{-3}$ & $2.2 \cdot 10^{21} \mathrm{~m}^{-3}$ \\
\hline $\begin{array}{l}\text { Reduction factor for } \\
\text { the space charge field }\end{array}$ & 0.56 & 0.28 \\
\hline
\end{tabular}

\section{Discussion}

Theoretical curves for the direct phase demodulation shown in Fig.2 were calculated using the vectorial system of the coupled wave equations (Eq.1). We found that this approach gives slightly better fitting than the analytical expression of Eq.12. The difference is more pronounced at higher electric fields and at smaller grating spacing. Its main reason is that the rigorous theory accounts for the 
polarization-state change due to the field-induced birefringence and optical activity of the crystal. Fractional intensity modulation calculated for the BTO crystal at $\Lambda=2 \mu \mathrm{m}$ using Eq. 1 and Eq.12 is shown in Fig. 6 by curves $a$ and $b$, respectively.

Much greater difference between the rigorous theory and an approximate analytical solution was revealed in the case of the PSM technique (recording under ac-field). Curves $c$ and $d$ in Fig. 6 show theoretical dependences of the fractional intensity modulation calculated for the BTO crystal at the same $\Lambda=2 \mu \mathrm{m}$ using Eq.1 (coupled wave equations) and Eq.7 (analytical expression), respectively. Note that the analytical solution was derived assuming $m(z)=$ constant [8], which is not the case even in the configuration of anisotropic diffraction with minimized energy exchange. Therefore, the analytical expression cannot be used for $m(0)<<$ 1.

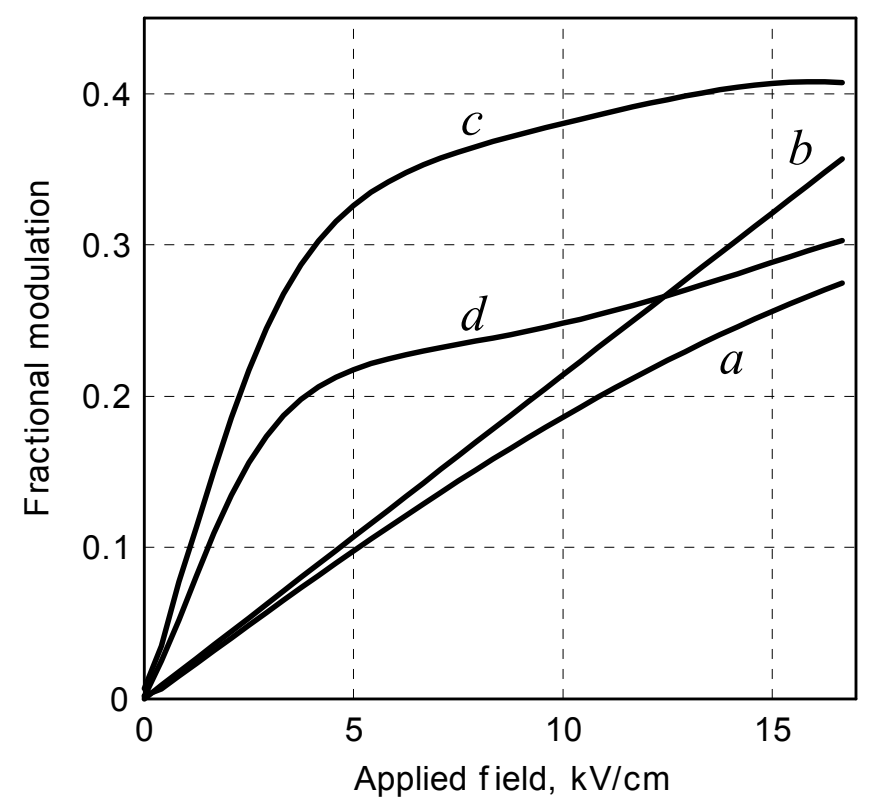

Figure 6. Comparison of the numerical solution of the coupled wave equations with analytical expression for direct phase demodulation (curves $a$ and $b$ ) and PSMtechnique (curves $c$ and $d$ ). All calculations were executed for BTO crystal at $\Lambda=2 \mu \mathrm{m}$ with $m(0)=0.1$ using the material parameters listed in the Table 1 .

In Fig.7 we summarize the modulus of the space-charge field calculated from the data obtained with different experimental techniques. Space-charge-field amplitude shown in Fig.7 was measured at $\Lambda=4 \mu \mathrm{m}$ for the BTO sample and at $\Lambda$ $=3.6 \mu \mathrm{m}$ for the GaP sample. Chosen grating spacing was certainly far from the optimal in the GaP crystal because of relatively small trap concentration. As one can see, $\left|E_{K}\right|$ generated under external ac-field is higher than that formed under dcfield. The physical reason of such extra-enhancement under ac-field is resonance excitation of space charge waves as it was suggested by Sturman et al. [15]. The maximum of the quality factor of the space-charge waves (calculated taking into account the reduction factor $f_{E}$ ) is 1.6 for BTO crystal and 0.26 for GaP crystal at $\Lambda=4$ and $3.6 \mu \mathrm{m}$, respectively. This difference in the quality factor explains smaller enhancement of $\left|E_{K}\right|$ under ac-field in the GaP sample.

Simultaneous fitting of all experimental data with the theory means that the relative influence of the applied field on the space-charge field is in complete 
accordance with predictions of the standard one-level model of the photorefractive effect. However, the absolute magnitude of $\left|E_{K}\right|$ is significantly reduced compare to that predicted by this model. Physical meaning of the introduced reduction factor is far from trivial. Our numerical simulation with coupled wave equations shows that possible presence of absorption grating (which is typical for BTO crystals [16]) practically does not change the magnitude of the reduction factor needed to fit the experiment with the theory. Moreover, recent analysis of the extended one-level model with two types of charge carriers shows that the real part of the space charge field created under dc-field is not generally decreased by the electron-hole competition factor [17]. Therefore, the space-charge-field reduction observed in the direct phase demodulation experiment could not be explained in the frame of the latter model, as well.

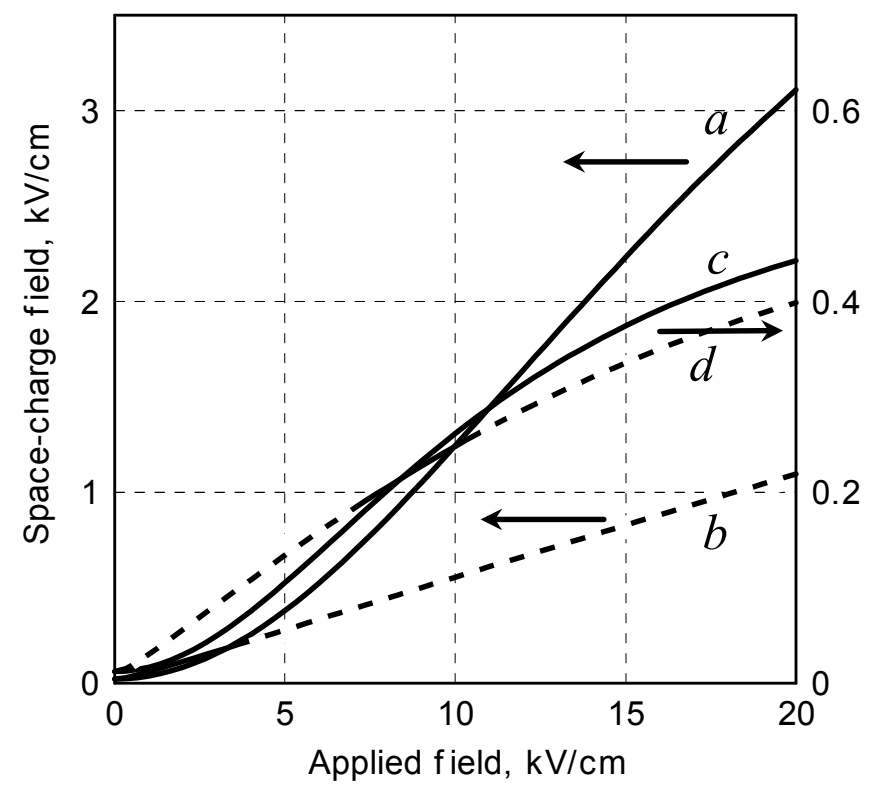

Figure 7. Space-charge-field amplitude as a function of the applied field for BTO at $\Lambda$ $=4 \mu \mathrm{m}$ (curves $a$ and $b$ ) and for GaP at $\Lambda=3.6 \mu \mathrm{m}$ (curves $c$ and $d$ ). Solid lines correspond to the non-local grating recording under ac-field and dashed lines - to the local recording under dc-field. All the data were obtained with the input intensity modultaion index $m(0)=0.1$. For better presentation the scale of the dependences for $\mathrm{GaP}$ is five times smaller (the right-hand scale).

At the moment we can propose a qualitative explanation of the observed reduction assuming a model with at least two different traps involved in the spacecharge-field formation. Population of the energetic levels associated with these centers may be a function of the light intensity because of different cross sections of both photons and electrons. Under certain conditions this may lead to diminishing of the spatially modulated term in the concentration of the excited free charges. Since the gradient of free charges is the only driving force for the spacecharge-field formation, its diminishing leads to the reduction of $\left|E_{K}\right|$. Note that similar model was recently applied for explanation of unusual recording dynamics in photorefractive $\mathrm{Bi}_{12} \mathrm{SiO}_{20}$ crystal [18]. Nevertheless, further elaboration of this and alternative model is strongly required. 


\section{Conclusion}

Three different experimental techniques (two-wave mixing gain, direct phase demodulation under dc-field, and phase demodulation with polarization filtration under ac-field) were applied for estimation of the space-charge-field amplitude in photorefractive crystals of $\mathrm{GaP}$ and $\mathrm{Bi}_{12} \mathrm{TiO}_{20}$. We found that all the experimental data can be simultaneously fitted with the theory in the frame of the standard onelevel model only if an additional reduction factor of the space charge field is introduced. However, the traditional one-level model of the photorefractive effect cannot explain appearance of this factor. These results show strong evidence that more than one photo-active level participate in the space-charge formation in fast photorefractive crystals.

\section{Acknowledgements}

This study is partly financed by European Commission under the Marie Curie Training Site Fellowship "Information Optics, new Tools and Application" (MCFH-1999-00840).

\section{References}

1. P. Delaye, A. Blouin, D. Drolet, L.-A. de Montmorillon, G. Roosen and J.-P. Monchalin, "Detection of ultrasonic motion of a scattering surface by photorefractive InP:Fe under an applied dc field", J. Opt. Soc. Am. B, 14 (1997), 1723-1732.

2. S. I. Stepanov and M. P. Petrov, "Efficient unstationary holographic recording in photorefractive crystals under an external alternating electric field", Opt. Comm. 53 (1985), 292-295.

3. G. A. Brost, "Photorefractive grating formations at large modulation with alternating electric fields", J. Opt. Soc. Am. B, 9 (1992), 1454-1460.

4. J. E. Millerd, E. M. Garmine, M. B. Klein, B. A. Wechsler, F. P. Strohkendl and G. A. Brost, "Photorefractive response at high modulation depths in BTO", J. Opt. Soc. Am. B, 9 (1992), 1449-1453.

5. A. A. Kamshilin, K. Päiväsaari, M. Klein and B. Pouet, "Adaptive interferometer using self-induced electro-optic modulation", Appl. Phys. Lett. 77 (2000), 4098-4100.

6. N. V. Kukhtarev, V. B. Markov, S. G. Odulov, M. S. Soskin, and V. L. Vinetskii, "Holographic storage in electrooptic crystals. I. Steady state", Ferroelectrics, 22 (1979), 949-960.

7. B. I. Sturman, E.V. Podivilov, K. H. Ringhofer, E. Shamonina, V. P. Kamenov, E. Nippolainen, V. V. Prokofiev and A. A. Kamshilin, "Theory of photorefractive vectorial wave coupling in cubic crystal", Phys. Rev. E, 60 (1999), 3332-3352.

8. K. Päiväsaari, A. A. Kamshilin, V. V. Prokofiev, B. Sturman, G. F. Calvo, M. Carrascosa and F. Agullo-Lopez, "Linear phase demodulation in photorefractive crystals with nonlocal response", J. Appl. Phys. 90 (2001), 3135-3141.

9. M. Z. Zha, P. Amrhein, and P. Günter, "Measurements of phase shift in photorefractive crystals by a novel method", IEEE J. Quant. Electr. 26 (1990) 788-792. 
10. P. Delaye, L. A. de Montmorillon and G. Rosen, "Transmission of time modulated optical signals through an absorbing photorefractive crystal", Opt. Comm. 118 (1995) 154-164.

11. P. Günter, "Holography, coherent light amplification and optical phase conjugation with photorefractive materials", Phys. Rep. 93 (1982), 199-299.

12. N. Nazhestkina, A. A. Kamshilin, O. V. Kobozev, V. V. Prokofiev, and T. Jääskeläinen, "Detection of small phase modulation using two-wave mixing in photorefractive crystals," Appl. Phys. B, 72 (2001) 767-773.

13. S. L. Sochava, E. V. Mokrushina, V. V. Prokofiev and S. I. Stepanov, "Experimental comparison of the ac field and the moving-grating holographicrecording techniques for $\mathrm{Bi}_{12} \mathrm{TiO}_{20}$ and $\mathrm{Bi}_{12} \mathrm{SiO}_{20}$ photorefractive crystals", J. Opt. Soc. Am. B, 10 (1993), 1600-1604.

14. K. Kuroda, Y. Okazaki, T Shimura, H. Okamura, M. Chihara, M. Itoh and I. Ogura, "Photorefractive effect in GaP", Opt. Lett. 15 (1990), 1197-1199.

15. B. I. Sturman, M. Mann, J. Otten, and K. H. Ringhofer, "Space-charge waves in photorefractive crystals and their parametric excitation," J. Opt. Soc. Am. B, 10 (1993), 1919-1932.

16. A. A. Kamshilin, "Simultaneous recording of absorption and photorefractive gratings in photorefractive crystals," Opt. Commun. 93 (1992) 350-358.

17. L-A. de Montmorillon, P. Delaye, J-C. Launay and G. Roosen, "Novel theoretical aspects on photorefractive ultrasonic detection and implementation of the sensor with an optimum sensitivity", J. Appl. Phys. 82 (1997), 59135922.

18. A. A. Kamshilin, O. V. Kobozev, A. I. Grachev and P. M. Karavaev, "Manifestation of long-lived photosensitivity gratings in two-wave mixing experiments with sillenite crystals", J. Opt. Soc. Am. B 19 (2002), 202-207. 